\title{
Influencia biomecánica de la artroplastia total de rodilla en el apoyo metatarsal
}

\author{
J. DOMINGO CEBOLLADA*, A. HERRERA RODRÍGUEZ*, \\ A. MARTÍNEZ MARTÍN*, J. PANISELLO SEBASTIA**, \\ J. MARTÍNEZ VILLA*, J.M. PÉREZ GARCÍA* \\ *Servicio de Cirugía Ortopédica y Traumatología \\ Hospital Miguel Servet \\ Zaragoza \\ **Servicio de Cirugía Ortopédica y Traumatología \\ Hospital de Alcañiz
}

\begin{abstract}
Resumen
Las articulaciones de la extremidad inferior forman una cadena cinemática y tanto desde el punto de vista normal como patológico se influyen mutuamente. El objetivo de nuestro trabajo es analizar las características del apoyo plantar en 100 pacientes subsidiarios de prótesis total de rodilla que presentan trastornos en la alineación del miembro antes y después de la corrección del eje de la extremidad que supone la cirugía. Además se compara el patrón de apoyo metatarsal con sujetos sanos de características antropométricas similares (grupo control).El genu varo preoperatorio se relaciona con un hiperapoyo de la paleta externa y un hipoapoyo de las paletas central e interna del antepié. En cambio el valgo fisiológico conseguido en el postoperatorio se corresponde con un patrón de apoyo metatarsal al año y a los dos años similar al del grupo control con pequeñas variaciones.
\end{abstract}

Palabras clave: Apoyo metatarsal, eje mecánico, baropodometría..

\section{Introducción}

Es necesario considerar el miembro inferior como una larga palanca sensible a las variaciones del equilibrio del pie. En estática y en dinámica, en las fases de apoyo, especialmente durante el periodo unipodal, todo el miembro inferior presenta un equilibrio frágil e inestable. Las condiciones del trabajo muscular varían en función de la acción plantar permanente, a partir del movimiento, del aumento o disminución de las palancas óseas, etc. Además, las articulaciones de la extremidad inferior forman una cadena cinemática y tanto desde el punto de vista normal como patológico se influyen mutuamente ${ }^{1}$.

El objetivo de nuestro trabajo es comparar las características del apoyo plantar en pacientes subsidiarios de artroplastia total de rodilla, antes y después de la intervención quirúrgica, con las de sujetos sanos de características antropométricas similares. Para ello debemos saber:

a) las presiones en los pacientes previas a la intervención quirúrgica. Tengamos en cuenta que la inmensa mayoría de estos sujetos presentan previamente a la cirugía trastornos importantes de la alineación de la extremidad inferior, casi siempre un eje mecánico de la misma varo.

b) las presiones de la superficie plantar en sujetos sanos y sin metatarsalgia, para determinar las presiones metatarsales que se pueden considerar como fisiológicas. Estos individuos constituirían el grupo control de nuestro estudio.

c) la presión metatarsal en los pacientes después de la intervención quirúrgica, la cual 
pretende la sustitución de las superficies articulares deterioradas por las protésicas así como un realineamiento de la extremidad y de esta forma un comportamiento biomecánico más adecuado de la misma.

El desarrollo de la baropodometría electrónica, especialmente en la última década ${ }^{2,3}$, permite cuantificar la distribución de presiones en la huella plantar de modo más exacto que con los métodos clásicos, así como la presión ejercida por cada metatarsiano y la situación del centro de presiones, mejorando así el estudio biomecánico de los efectos de la cirugía protésica de cadera y rodilla.

\section{Material y métodos}

Hemos realizado un estudio baropodométrico utilizando la plataforma PDS-93 ${ }^{4}$. Se trata de un sistema basado en sensores piezoresistivos que permite la adquisición, análisis y registro de presiones bajo la superficie plantar, con el objeto de calcular un mapa de presiones (cartografía plantar) que se corresponde a la presión ejercida en cada punto del pie sobre una plataforma plana, en posición estática y erecta. La sencillez de manejo del equipo y su reducido coste permite su utilización no sólo en los laboratorios de investigación, sino también en los Servicios y consultas diarias de Ortopedia. La forma de representación de las diferentes presiones y su distribución usa un código de colores, que puede ser definido por el usuario tanto en rango como en color asociado. De esta forma, el uso del color ayuda a una identificación rápida de los valores de presiones en el que nos movemos, teniendo una representación cartográfica de la carga en los diferentes puntos de la huella plantar. En la Figura 1 se muestra un aspecto global del entorno.

El grupo control del trabajo estaba integrado por 150 individuos sanos, sin patología del aparato locomotor ni dismetría, deformidades o desalineaciones en las extremidades inferiores.

El grupo patológico estaba constituido por 100 pacientes subsidiarios de artroplastia total de rodilla controlados en consultas externas de nuestro hospital y que fueron sometidos a la intervención quirúrgica entre junio de 1995 y septiembre de 1996.

Cada individuo perteneciente al grupo patológico ha sido evaluado en 5 ocasiones: preoperatoriamente, postoperatoriamente a los 3,6 y 12 meses, y por último un control postoperatorio realizado como media a los 24 meses desde la intervención, constituyéndose así 5 grupos de estudio para estudiar la evolución del apoyo metatarsal.

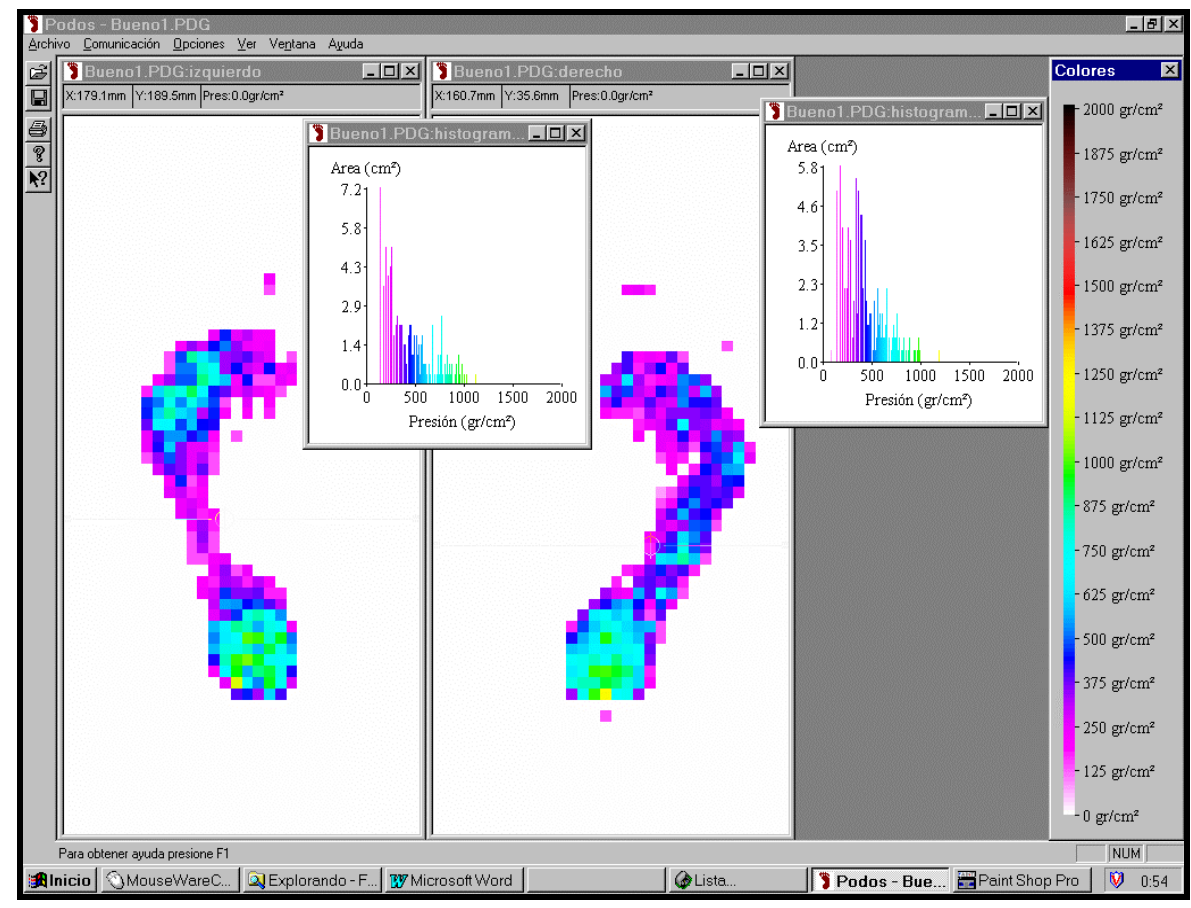

Figura 1. Registro de presiones bajo la superficie plantar. 
En el podoscopio se analizaron las huellas de cada pie en apoyo bipodal, tanto cada pie por separado como los dos pies juntos sobre la plataforma.

Además, en el postoperatorio se han agrupado los pacientes según el valor del ángulo femorotibial anatómico, constituyendo dos grupos: el primero considerado como de corrección suficiente con valores comprendidos de $4^{\circ}$ a $10^{\circ}$ de valgo y el segundo considerado como de corrección insuficiente con valores comprendidos de genu varo a $3^{\circ}$ de valgo para comparar las características del apoyo plantar según la realineación de la extremidad conseguida.

La presión correspondiente a cada metatarsiano se ha recogido en $\mathrm{Kg} . / \mathrm{cm}^{2}$ y en porcentaje relativo a la presión ejercida en el antepié. Tanto en el grupo control como en el patológico cada registro se ha repetido en todos los sujetos cinco veces hasta conseguir una huella uniforme en el baropodómetro electrónico, ya que la huella puede variar dependiendo de la carga ejercida por el sujeto o de las contracciones de los músculos plantares ${ }^{3,5}$.

Tras el estudio de la normalidad de la distribución realizado usando el test de Kolmogorov-Smirnov hemos concluido que la distribución de la población base de nuestro estudio no es normal, por tanto, hemos utilizado test no paramétricos. Con los datos obtenidos se ha creado una base de datos, procesando los resultados por medio del paquete estadístico SPSS.

\section{Resultados}

En el grupo control se han realizado 300 registros correspondientes a 150 individuos, 48 varones $(32 \%)$ y 102 mujeres $(68 \%)$, con edades comprendidas entre 44 y 65 años (edad media 57,9 años). La carga puntual de la cabeza de los metatarsianos registra un predominio de la carga central siendo el metatarsiano que mayor carga soporta el tercero. En concreto los valores en porcentaje de carga registrados fueron $21,46 \%$ para la cabeza del segundo, $24,65 \%$ para el tercero y $20,10 \%$ para el cuarto, con valores menores de presión para los metatarsianos periféricos, de $19,36 \%$ para el primero y $14,43 \%$ para el quinto metatarsiano.

El ángulo femorotibial anatómico en estos sujetos registró un valor medio de $7,83^{\circ}$ de valgo. Las coordenadas del centro de presión lo situaban en posición externa en relación con el eje mecánico del pie en 27 registros (9\%), posición central en
258 registros $(86 \%)$ y situación medial o interna en 15 registros $(5 \%)$.

En el grupo patológico se han realizado registros a 100 individuos (300 registros en cada control clínico efectuado, un total de 1500 registros) subsidiarios de artroplastia total de rodilla, 26 varones y 74 mujeres, con una edad media de 69,11 años (mínimo 54 y máximo 83 años). Los diagnósticos registrados en nuestros pacientes han sido Gonartrosis (94 de los 100 casos), Artritis Reumatoide (3 casos) y Necrosis Condíleas (3 casos). En 91 pacientes existía un genu varo previo. En el $75 \%$ de los casos la afectación era bilateral.

La situación del centro de presión, obtenida con el baropodómetro electrónico, correspondiente a los controles previo y postoperatorios realizados, se muestra en la Tabla 1 para el pie de la extremidad operada y en la Tabla 2 para el pie de la pierna no operada.

Puede apreciarse un predominio externo (63\%) de las coordenadas del centro de presión en el pie del lado afecto, en el registro preoperatorio, que gradualmente se medializa en los controles postoperatorios hasta alcanzar en un $84 \%$ la posición central en el último control a los dos años de la intervención. En el pie de la extremidad no intervenida existen pocas variaciones, situándose el centro de presión centrado mayoritariamente $(68 \%$ $-74 \%$ ) y en posición externa entre un $24 \%$ y $31 \%$.

En la Tabla 3 se reflejan los valores medios de los ángulos femorotibiales anatómico y mecánico, medidos en el preoperatorio y en el postoperatorio. Las medidas negativas corresponden a ángulos de genu valgo. En nuestro estudio el valor medio del ángulo femorotibial anatómico preoperatorio ha sido de $6,09^{\circ}$ de varo llegándose tras la corrección quirúrgica a una media de $5,53^{\circ}$ de valgo siendo estas diferencias significativas $(\mathrm{p}<0,001)$.

En la Tabla 4A se muestra la comparación mediante el test de Wilcoxon, de los valores medios de porcentaje de carga, a nivel de cada metatarsiano correspondientes al pie de la extremidad inferior intervenida, antes y después de la colocación de la prótesis de rodilla. Existen diferencias significativas para todos los metatarsianos, que se mantienen en los sucesivos controles efectuados.

En la extremidad no intervenida (Tabla 4B) no existen diferencias significativas para ningún metatarsiano con respecto a los valores preoperatorios, manteniéndose este estado en todos los controles. 
Tabla 1. Situación del centro de presión en el pie del lado operado (MIP) para cada uno de los registros previo y postoperatorio

\begin{tabular}{|l|c|c|}
\hline Variable cualitativa analizada & Frecuencia & Porcentaje \\
\hline Centro de presión MIP inicial & & \\
\hline Central & 35 & 35,0 \\
Medial & 2 & 2,0 \\
Externo & 63 & 63,0 \\
\hline Centro de presión MIP 3 meses & & \\
\hline Central & 73 & 73,0 \\
Medial & 22 & 22,0 \\
Externo & 5 & 5,0 \\
\hline Centro de presión MIP 6 meses & 75 & 75,0 \\
\hline Central & 19 & 19,0 \\
Medial & 6 & 6,0 \\
Externo & & 82,0 \\
\hline Centro de presión MIP 1 año & 82 & 12,0 \\
\hline Central & 12 & 6,0 \\
Medial & 6 & 84,0 \\
Externo & & 11,0 \\
\hline Centro de presión MIP 2 años & 84 & 5,0 \\
\hline Central & 11 & \\
Medial & 5 & \\
Externo & & \\
\hline
\end{tabular}

Tabla 2. Situación del centro de presión en el pie del lado no operado (MIN) para cada uno de los registros previo y postoperatorio.

\begin{tabular}{|l|c|c|}
\hline Variable cualitativa analizada & Frecuencia & Porcentaje \\
\hline Centro de presión MIN inicial & & \\
\hline Central & 71 & 71,0 \\
Medial & 1 & 1,0 \\
Externo & 28 & 28,0 \\
\hline Centro de presión MIN 3 meses & & \\
\hline Central & 68 & 68,0 \\
Medial & 1 & 1,0 \\
Externo & 31 & 31,0 \\
\hline Centro de presión MIN 6 meses & & 75,0 \\
\hline Central & 75 & 25,0 \\
Externo & 25 & \\
\hline Centro de presión MIN 1 año & & 74,0 \\
\hline Central & 74 & 26,0 \\
Externo & 26 & 76,0 \\
\hline Centro de presión MIN 2 años & & 24,0 \\
\hline Central & 76 & \\
Externo & 24 & \\
\hline
\end{tabular}


Tabla 3. Descripción de las variables cuantitativas incluidas en el grupo patológico.

\begin{tabular}{|l|c|c|c|c|}
\hline \multicolumn{1}{|c|}{ Variable cuantitativa } & Media & $\begin{array}{c}\text { Desviación } \\
\text { Estándar }\end{array}$ & Mínimo & Máximo \\
\hline Angulo femoral mecánico-anatómico & 6,51 & 0,77 & 5,00 & 8,00 \\
Angulo femorotibial anatómico preoperatorio & 6,09 & 8,41 & $-28,00$ & 22,00 \\
Angulo femorotibial anatómico postoperatorio & $-5,53$ & 2,75 & $-10,00$ & 5,00 \\
Angulo femorotibial mecánico preoperatorio & 13,78 & 6,48 & $-18,00$ & 29,00 \\
Angulo femorotibial mecánico postoperatorio & 0,99 & 2,78 & $-3,00$ & 12,00 \\
\hline
\end{tabular}

Tabla 4A: Comparación de medias del porcentaje de carga metatarsal, entre el registro previo y los postoperatorios a los 3 meses, 6 meses, 1 año y 2 años, en el miembro inferior operado

\begin{tabular}{|c|c|c|c|c|c|}
\hline Registro & M1 & M2 & M3 & M4 & M5 \\
\hline $\begin{array}{l}\text { Preoperatorio } \\
\mathrm{p}\end{array}$ & $\begin{array}{r}\mathbf{1 9 , 0 2} \pm \mathbf{1 0 , 2} \\
0,0000 * * *\end{array}$ & $\begin{array}{r}\mathbf{1 9 , 0 7} \pm \mathbf{5 , 6 9} \\
0,0010 * *\end{array}$ & $\begin{array}{c}\mathbf{1 9 , 7 4} \pm \mathbf{5 , 4 5} \\
0,1427\end{array}$ & $\begin{array}{r}\mathbf{2 2 , 4 8} \pm \mathbf{8 , 1 7} \\
0,0000 * * *\end{array}$ & $\begin{array}{r}\mathbf{1 9 , 7 0} \pm \mathbf{7 , 7 3} \\
0,0003 * * *\end{array}$ \\
\hline Pos (3 m) & $24,27 \pm 7,63$ & $21,03 \pm 4,28$ & $21,00 \pm 5,05$ & $17,40 \pm 4,34$ & $16,30 \pm 5,22$ \\
\hline $\begin{array}{l}\text { Preoperatorio } \\
\text { p } \\
\text { Pos }(6 \mathbf{~ m}) \\
\end{array}$ & $\begin{array}{c}19,02 \pm 10,2 \\
0,0009 * * * \\
\mathbf{2 2 , 1 7} \pm \mathbf{7 , 8 0}\end{array}$ & $\begin{array}{l}19,07 \pm 5,69 \\
0,0000 * * * \\
\mathbf{2 1 , 7 2} \pm \mathbf{4 , 5 0}\end{array}$ & $\begin{array}{c}19,74 \pm 5,45 \\
0,0000 * * * \\
\mathbf{2 3 , 0 6} \pm \mathbf{3 , 8 4}\end{array}$ & $\begin{array}{c}22,48 \pm 8,17 \\
0,0000 * * * \\
\mathbf{1 7 , 4 1} \pm \mathbf{4 , 0 0}\end{array}$ & $\begin{array}{c}19,70 \pm 7,73 \\
0,0000 * * * \\
\mathbf{1 5 , 6 5} \pm \mathbf{5 , 4 6}\end{array}$ \\
\hline $\begin{array}{l}\text { Preoperatorio } \\
\text { p } \\
\text { Pos }(\mathbf{1} \text { año })\end{array}$ & $\begin{array}{r}19,02 \pm 10,2 \\
0,0009 * * \\
\mathbf{2 1 , 6 7} \pm \mathbf{7 , 1 1}\end{array}$ & 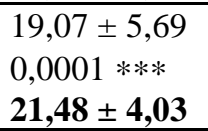 & $\begin{array}{c}19,74 \pm 5,45 \\
0,0000 * * * \\
\mathbf{2 3 , 9 6} \pm \mathbf{4 , 5 2}\end{array}$ & $\begin{array}{c}22,48 \pm 8,17 \\
0,0000 * * * \\
\mathbf{1 7 , 6 5} \pm \mathbf{3 , 5 5}\end{array}$ & $\begin{array}{c}19,70 \pm 7,73 \\
0,0000 * * * \\
\mathbf{1 5 , 2 4} \pm \mathbf{4 , 4 4}\end{array}$ \\
\hline $\begin{array}{l}\text { Preoperatorio } \\
\text { p } \\
\text { Pos (2 años })\end{array}$ & $\begin{array}{r}19,02 \pm 10,2 \\
0,0019 * * \\
\mathbf{2 1 , 1 0} \pm \mathbf{6 , 6 6}\end{array}$ & 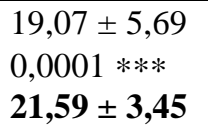 & $\begin{array}{c}19,74 \pm 5,45 \\
0,0000 * * * \\
\mathbf{2 4 , 6 7} \pm \mathbf{3 , 2 2}\end{array}$ & $\begin{array}{c}22,48 \pm 8,17 \\
0,0000 * * * \\
\mathbf{1 8 , 0 6} \pm \mathbf{3 , 1 3}\end{array}$ & $\begin{array}{c}19,70 \pm 7,73 \\
0,0000 * * * \\
\mathbf{1 4 , 5 7} \pm \mathbf{4 , 0 5}\end{array}$ \\
\hline
\end{tabular}

Tabla 4B: Comparación de medias del porcentaje de carga metatarsal, entre el registro previo y los postoperatorios a los 3 meses, 6 meses, 1 año y dos años, en el miembro inferior no operado

\begin{tabular}{|c|c|c|c|c|c|}
\hline Registro & M1 & M2 & M3 & M4 & M5 \\
\hline $\begin{array}{c}\text { Preoperatorio } \\
\text { p } \\
\text { Pos }(3 \mathrm{~m})\end{array}$ & $\begin{array}{c}\mathbf{1 9 , 8 7} \pm \mathbf{7 , 5 9} \\
0,4582 \\
\mathbf{1 9 , 8 1} \pm \mathbf{7 , 9 6}\end{array}$ & $\begin{array}{c}21, \mathbf{6 1} \pm \mathbf{5 , 2 1} \\
0,0980 \\
\mathbf{2 1 , 2 3} \pm \mathbf{6 , 6 7}\end{array}$ & $\begin{array}{c}22,05 \pm \mathbf{4 , 9 8} \\
0,5036 \\
\mathbf{2 1 , 9 0} \pm \mathbf{4 , 1 2}\end{array}$ & $\begin{array}{c}19,68 \pm \mathbf{4 , 7 8} \\
0,7163 \\
\mathbf{1 9 , 6 0} \pm \mathbf{5 , 2 3}\end{array}$ & $\begin{array}{c}\mathbf{1 6 , 7 9} \pm \mathbf{5 , 7 9} \\
0,2027 \\
\mathbf{1 7 , 4 6} \pm \mathbf{5 , 4 5}\end{array}$ \\
\hline $\begin{array}{c}\text { Preoperatorio } \\
\text { p } \\
\text { Pos }(6 \mathbf{~ m}) \\
\end{array}$ & $\begin{array}{c}19,87 \pm 7,59 \\
0,3131 \\
\mathbf{1 9 , 4 7} \pm \mathbf{7 , 6 5}\end{array}$ & $\begin{array}{c}21,61 \pm 5,21 \\
0,2494 \\
\mathbf{2 1 , 3 0} \pm \mathbf{5 , 4 4}\end{array}$ & $\begin{array}{c}22,05 \pm 4,98 \\
0,5328 \\
\mathbf{2 2 , 5 3} \pm \mathbf{4 , 6 7}\end{array}$ & $\begin{array}{c}19,68 \pm 4,78 \\
0,9015 \\
\mathbf{1 9 , 4 7} \pm \mathbf{4 , 8 0}\end{array}$ & $\begin{array}{c}16,79 \pm 5,79 \\
0,5565 \\
\mathbf{1 7 , 2 2} \pm \mathbf{4 , 9 7}\end{array}$ \\
\hline $\begin{array}{c}\text { Preoperatorio } \\
\text { p } \\
\text { Pos (1 año) } \\
\end{array}$ & $\begin{array}{c}19,87 \pm 7,59 \\
0,4697 \\
\mathbf{1 9 , 6 8} \pm \mathbf{7 , 6 0} \\
\end{array}$ & $\begin{array}{c}21,61 \pm 5,21 \\
0,3570 \\
\mathbf{2 1 , 0 3} \pm \mathbf{4 , 6 4}\end{array}$ & $\begin{array}{c}22,05 \pm 4,98 \\
0,6412 \\
\mathbf{2 2 , 2 1} \pm \mathbf{4 , 3 7}\end{array}$ & $\begin{array}{c}19,68 \pm 4,78 \\
0,7492 \\
\mathbf{1 9 , 8 2} \pm \mathbf{4 , 8 7} \\
\end{array}$ & $\begin{array}{c}16,79 \pm 5,79 \\
0,2539 \\
\mathbf{1 7 , 2 6} \pm \mathbf{5 , 7 0}\end{array}$ \\
\hline $\begin{array}{c}\text { Preoperatorio } \\
\text { p } \\
\text { Pos (2 años) }\end{array}$ & $\begin{array}{c}19,87 \pm 7,59 \\
0,5101 \\
\mathbf{1 9 , 8 8} \pm \mathbf{7 , 1 5}\end{array}$ & $\begin{array}{c}21,61 \pm 5,21 \\
0,6380 \\
\mathbf{2 1 , 1 3} \pm \mathbf{5 , 4 6}\end{array}$ & $\begin{array}{c}22,05 \pm 4,98 \\
0,4930 \\
\mathbf{2 2 , 7 6} \pm \mathbf{5 , 2 5}\end{array}$ & $\begin{array}{c}19,68 \pm 4,78 \\
0,4369 \\
\mathbf{2 0 , 1 1} \pm \mathbf{3 , 5 2}\end{array}$ & $\begin{array}{c}16,79 \pm 5,79 \\
0,9678 \\
\mathbf{1 6 , 1 1} \pm \mathbf{6 , 0 9}\end{array}$ \\
\hline
\end{tabular}


Estudio mediante el test de Mann-Whitney de la relación entre la carga y el eje femorotibial anatómico conseguido en el postoperatorio.

Se han agrupado los pacientes según el valor del eje anatómico femorotibial, constituyendo dos grupos: el primero considerado como de corrección suficiente con valores comprendidos de $4^{\circ}$ a $10^{\circ}$ de valgo y el segundo considerado como de insuficiente corrección con valores comprendidos de genu varo a $3^{\circ}$ de valgo.

En la Tabla 5 puede apreciarse la distribución de la carga en ambos grupos, mostrando una tendencia similar en los dos grupos a nivel de los metatarsianos. Se produce un aumento inmediato de la carga, tras la intervención quirúrgica, en el primer registro para los tres primeros metatarsianos (M1, M2, M3) y un descenso para los dos últimos (M4, M5). En los controles sucesivos del postoperatorio la carga aumenta progresivamente para los metatarsianos centrales (M2, M3, M4) y disminuye para los laterales (M1, M5). Las diferencias más pronunciadas en los primeros controles, de esta forma, tienden a desaparecer en los últimos registros de la evolución clínica (1 año y 2 años).

Sólo existen diferencias significativas entre los dos grupos, considerando los controles en el postoperatorio, para los registros del cuarto metatarsiano (6 meses y 1 año) y quinto (3 meses).

Tabla 5. Comparación de los valores medios del porcentaje de carga de los metatarsianos en los sucesivos registros entre los pacientes en que se consigue una alineación correcta de la extemidad y aquellos en los que la corrección es insuficiente en la extremidad intervenida.

\begin{tabular}{|c|c|c|c|c|}
\hline META & REGISTROS & I - VALGO & II - VARO & (p) \\
\hline M1 & $\begin{array}{c}\text { PREOPER. } \\
3 \text { MESES } \\
6 \text { MESES } \\
1 \text { AÑO } \\
\text { 2 AÑNOS }\end{array}$ & $\begin{array}{l}\mathbf{1 9 , 2 9} \pm \mathbf{9 , 9 1} \\
23,86 \pm 7,64 \\
21,16 \pm 7,18 \\
21,13 \pm 6,85 \\
\mathbf{2 0 , 6 8} \pm \mathbf{9 , 3 5}\end{array}$ & $\begin{array}{l}\mathbf{1 7 , 6 7} \pm \mathbf{1 1 , 6} \\
26,31 \pm 7,47 \\
24,46 \pm 8,99 \\
24,28 \pm 7,99 \\
\mathbf{2 2 , 5 6} \pm \mathbf{1 0 , 2}\end{array}$ & $\begin{array}{l}\mathbf{0 , 2 3 6 4} \\
0,2363 \\
0,1266 \\
0,1175 \\
\mathbf{0 , 4 4 0 1} \\
\end{array}$ \\
\hline M2 & $\begin{array}{c}\text { PREOPER. } \\
3 \text { MESES } \\
6 \text { MESES } \\
1 \text { AÑO } \\
\text { 2 A ANOS }\end{array}$ & $\begin{array}{l}\mathbf{1 9 , 6 4} \pm \mathbf{5 , 7 4} \\
20,91 \pm 4,36 \\
21,82 \pm 4,44 \\
21,30 \pm 3,98 \\
\mathbf{2 1 , 7 3} \pm \mathbf{4 , 3 9}\end{array}$ & $\begin{array}{l}\mathbf{1 6 , 2 4} \pm \mathbf{4 , 6 1} \\
21,62 \pm 3,92 \\
21,21 \pm 4,85 \\
22,35 \pm 4,30 \\
\mathbf{2 1 , 6 2} \pm \mathbf{4 , 8 9}\end{array}$ & $\begin{array}{l}\mathbf{0 , 0 1 3 7} * \\
0,5415 \\
0,6628 \\
0,4215 \\
\mathbf{0 , 7 7 6 5}\end{array}$ \\
\hline M3 & $\begin{array}{c}\text { PREOPER. } \\
3 \text { MESES } \\
6 \text { MESES } \\
1 \text { AÑ } \\
\text { 2 A ANOS } \\
\end{array}$ & $\begin{array}{l}\mathbf{2 0 , 1 1} \pm \mathbf{5 , 3 8} \\
20,96 \pm 5,19 \\
23,18 \pm 4,04 \\
23,94 \pm 4,72 \\
\mathbf{2 4 , 3 6} \pm \mathbf{4 , 1 7} \\
\end{array}$ & $\begin{array}{l}\mathbf{1 7 , 9 7} \pm \mathbf{5 , 6 1} \\
21,24 \pm 4,42 \\
22,44 \pm 2,66 \\
24,06 \pm 3,48 \\
\mathbf{2 5 , 6 0} \pm \mathbf{4 , 9 5} \\
\end{array}$ & $\begin{array}{l}\mathbf{0 , 0 9 0 4} \\
0,8007 \\
0,4379 \\
0,5353 \\
\mathbf{0 , 4 4 9 5} \\
\end{array}$ \\
\hline M4 & $\begin{array}{c}\text { PREOPER. } \\
3 \text { MESES } \\
6 \text { MESES } \\
1 \text { AÑ } \\
\text { 2 A ANOS } \\
\end{array}$ & $\begin{array}{r}\mathbf{2 2 , 1 9} \pm \mathbf{8 , 1 4} \\
17,56 \pm 4,16 \\
17,79 \pm 3,98 \\
18,01 \pm 3,64 \\
\mathbf{1 8 , 7 3} \pm \mathbf{3 , 9 4} \\
\end{array}$ & $\begin{array}{r}\mathbf{2 3 , 8 9} \pm \mathbf{8 , 4 5} \\
16,60 \pm 5,19 \\
15,54 \pm 3,62 \\
15,86 \pm 2,48 \\
\mathbf{1 6 , 8 0} \pm \mathbf{3 , 4 9} \\
\end{array}$ & $\begin{array}{l}\mathbf{0 , 2 8 5 0} \\
0,4516 \\
0,0195 * \\
0,0276 * \\
\mathbf{0 , 2 0 1 9} \\
\end{array}$ \\
\hline M5 & $\begin{array}{c}\text { PREOPER. } \\
3 \text { MESES } \\
6 \text { MESES } \\
1 \text { AÑO } \\
\text { 2 AÑNOS }\end{array}$ & $\begin{array}{r}\mathbf{1 8 , 7 7} \pm \mathbf{7 , 3 0} \\
16,72 \pm 5,25 \\
16,04 \pm 5,57 \\
15,61 \pm 4,65 \\
\mathbf{1 4 , 5 7} \pm \mathbf{5 , 5 0}\end{array}$ & $\begin{array}{l}\mathbf{2 4 , 2 2} \pm \mathbf{8 , 4 0} \\
14,22 \pm 4,70 \\
13,75 \pm 4,53 \\
13,43 \pm 2,70 \\
\mathbf{1 3 , 4 1} \pm \mathbf{4 , 3 1}\end{array}$ & $\begin{array}{l}\mathbf{0 , 0 1 3 4} * \\
0,0449 * \\
0,0611 \\
0,0555 \\
\mathbf{0 , 5 5 9 9}\end{array}$ \\
\hline
\end{tabular}




\section{Discusión}

Son numerosos los trabajos que han relacionado una correcta alineación de la extremidad sometida a artroplastia total de rodilla con la mayor o menor durabilidad del implante debido al desgaste de sus componentes, etc.

En 1988 Hsu $^{6}$ estudia en sujetos sanos la geometría de la articulación de la rodilla, hallando un valor medio del ángulo femorotibial mecánico de $1,2^{\circ}$ de varo sin encontrar diferencias significativas entre ambos sexos, y en cuanto al ángulo femoral mecánico-anatómico, la media encontrada fue de $4,9^{\circ}$. Otros autores han encontrado valores similares ${ }^{7,8}$. Concluye que existe correlación entre la alineación femorotibial y el porcentaje de carga soportada por el platillo medial de la articulación, aumentando ésta a medida que aumenta el varo de la extremidad, por lo que remarca la importancia de obtener una correcta alineación de la extremidad de cara a la obtención de buenos resultados en cirugía de rodilla.

Tew ${ }^{9}$ admite el postulado general de que un correcto alineamiento de la extremidad conseguido en el postoperatorio, es recomendable de cara al éxito de la artroplastia total de rodilla pero señala que en un porcentaje no despreciable de casos, un alineamiento incorrecto no conlleva el aflojamiento de los componentes del implante y viceversa, en algunos casos con buen alineamiento se producen fracasos de la prótesis.

Por otra parte, en algunos casos en los que en el postoperatorio inicial se consigue un eje correcto de la extremidad intervenida, con el tiempo se producen deformidades en varo o en valgo, en estos casos la tasa de aflojamientos es incluso mayor que en aquellos que presentan mala alineación desde la intervención quirúrgica. El autor remarca que además de una alineación correcta otros factores deben ser considerados como posibles causas de aflojamientos asépticos (inadecuado balance músculoligamentoso, etc.), además de que es muy difícil establecer en cada caso particular cuáles son los límites de un alineamiento adecuado. Otros autores comparten estos criterios ${ }^{10,11}$.

En nuestro estudio, el valor medio del ángulo femorotibial anatómico preoperatorio ha sido de $6,09^{\circ}$ de varo, llegándose tras la corrección quirúrgica a una media de $5,53^{\circ}$ de valgo. Las diferencias entre estos valores son lógicamente significativas y se relacionan con diferentes patrones de apoyo metatarsal pre y postoperatorio registrados en la extremidad intervenida y ya comentados, en cuanto a distribución de cargas metatarsales y situación del centro de presiones. En resumen, podemos decir que en la inmensa mayoría de los casos de nuestro estudio el genu varo preoperatorio se relaciona con un hiperapoyo de la paleta externa y un hipoapoyo de las paletas central e interna del antepié, lo que concuerda con el alto porcentaje de localizaciones externas del centro de presiones de la huella plantar. En cambio el valgo fisiológico conseguido en el postoperatorio se corresponde con un patrón de apoyo metatarsal, en cuanto a las cargas de cada metatarsiano y situación del centro de presiones, al año y a los dos años similar al del grupo control con pequeñas variaciones. La representación gráfica de la distribución espacial de la carga a nivel de los metatarsianos en el grupo control y el grupo con patología tratado mediante artroplastia total de rodilla, mostrada en la Figuras 2 y 3 , ayuda a ver el efecto del tratamiento mencionado. Los valores registrados a los dos años de la intervención quedan bien aglutinados dentro de la distribución espacial del grupo control, considerando en cada eje el porcentaje de carga del primero, tercero y quinto metatarsianos respectivamente, como representación de las tres paletas biomecánicas. Esto se debe a la redistribución progresiva de las cargas metatarsales en el antepié que se ha producido en los pacientes tras la realineación conseguida tras la cirugía.

En los primeros registros, especialmente a los tres meses, las variaciones son mayores debido a la existencia de otros factores ya comentados (balance muscular, partes blandas, etc.) y que reflejan el proceso de adaptación del paciente al implante colocado. En este sentido, Andriacchi 12, 13 defiende que el correcto balance entre las cargas soportadas por el compartimento medial y lateral de la rodilla no sólo se relaciona con el alineamiento, sino también con la presencia de una buena función de la musculatura y una suficiente tensión en las partes blandas para contrarrestar los momentos de aducción generados durante la bipedestación y marcha normales. Si hay demasiada laxitud la carga se transmite casi totalmente al compartimento medial de la rodilla incluso en casos con buen alineamiento lo que puede repercutir en un aflojamiento protésico precoz. 


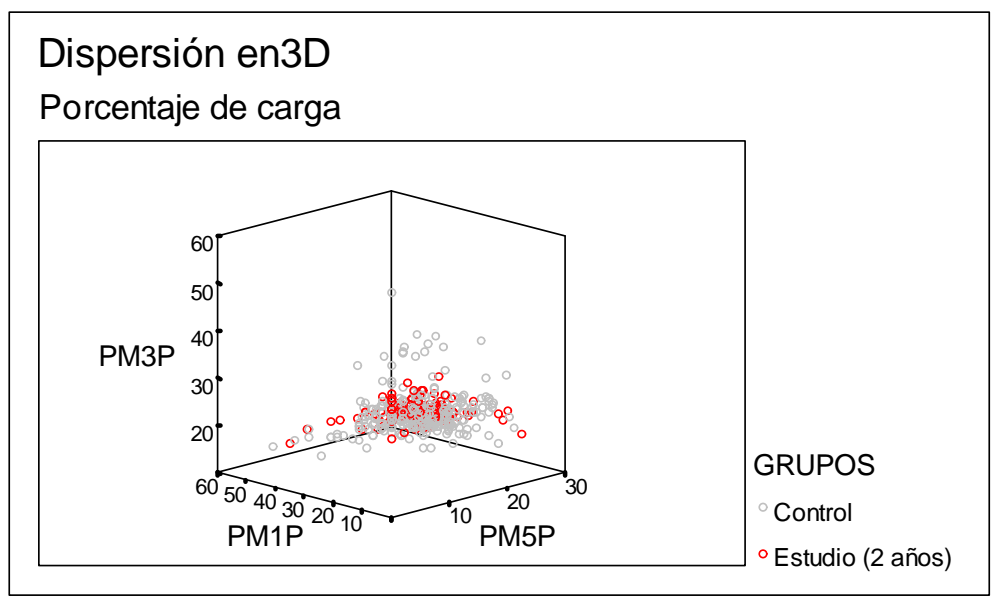

Figura 2. Distribución espacial de carga a nivel de metatarsianos para el grupo control y el grupo con artroplastia total de rodilla

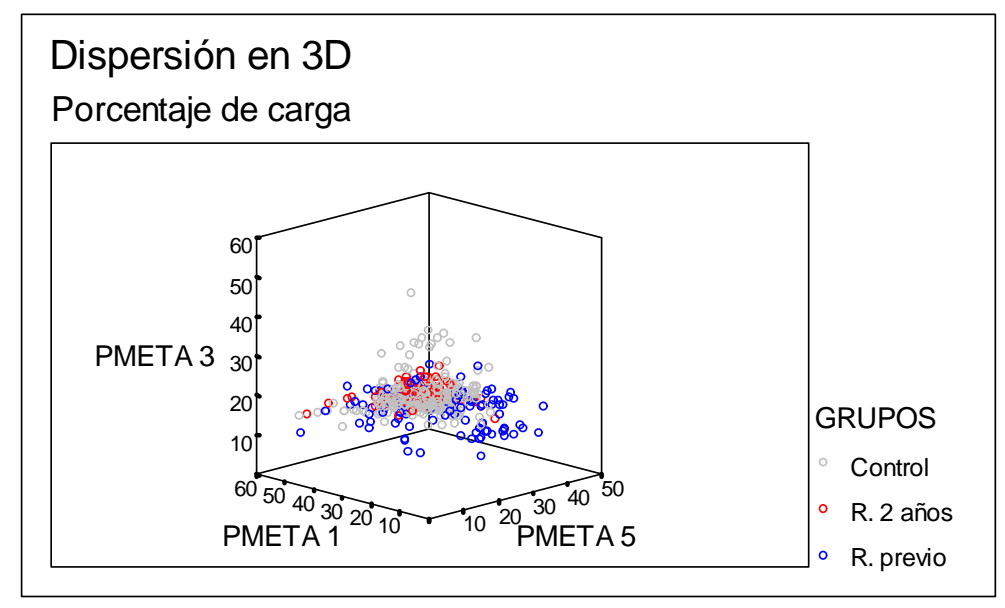

Figura 3. Distribución espacial de carga a nivel de metatarsianos para el grupo control y el grupo con artroplastia total de rodilla

También nos ha parecido interesante la comparación de la distribución de cargas metatarsales postoperatoria entre el grupo de pacientes en que se ha conseguido una alineación correcta de la extremidad $\left(4^{\circ}-10^{\circ}\right.$ de valgo $)$ y el resto de sujetos, de cara a averiguar si una alineación incorrecta o insuficiente se relaciona con cambios significativos en el apoyo plantar. No hemos encontrado diferencias en este sentido, incluso en algún caso se ha registrado una tendencia (sin llegar a la significación estadística) inversa a la esperada, con pacientes que presentaban una escasa corrección del varo preoperatorio y sin embargo mayor apoyo en la paleta interna del antepié que los casos con eje en valgo correcto.

Esto puede deberse a que estos casos correspondían a las mayores deformidades en varo preoperatorias (generalmente más de $20^{\circ}$ de ángulo femorotibial anatómico), con lo que aunque no llegaban a alcanzar un eje entre $4-10^{\circ}$ de valgo, el número de grados corregido sí era muy importante y probablemente conseguido a expensas de una liberación extensa de partes blandas, con la consiguiente existencia en estos casos de cierta debilidad en el compartimento interno de la rodilla que podría explicar este hallazgo, como señalan algunos autores ${ }^{14}$. Por otra parte, es importante señalar que tras la brusca redistribución de las presiones en el antepié observada en el primer control postoperatorio, con aumento de la carga soportada por los tres primeros metatarsianos y disminución de la soportada por el cuarto y quinto, se observa posteriormente una tendencia en los 
sucesivos controles postoperatorios de aumento de la carga en los metatatarsianos centrales (M2, M3 y M4) y disminución en los laterales (M1 y M5), con lo que las diferencias entre los dos grupos tienden a desaparecer en los últimos controles (al año y a los dos años). Sin embargo, el escaso número de casos en los que no se logra el valgo fisiológico en el postoperatorio en nuestro estudio, no nos permite establecer conclusiones definitivas en este sentido.

Por último, en la extremidad no intervenida, los valores registrados en cuanto a la distribución de cargas y situación del centro de presiones, siempre difieren un poco de unos registros a otros aunque sin diferencias significativas. Este hecho está en consonancia con la idea del pie como estructura dinámica sujeto a constantes variaciones "fisiológicas" del apoyo. En este sentido Smith ${ }^{15}$ habla del "dynamic standing" frente al "static standing”.

\section{Conclusiones}

1. Los individuos sin trastornos en la alineación de la extremidad inferior presentan un patrón de apoyo metatarsal caracterizado por un predominio central de la carga, siendo la zona que mayor presión soporta la cabeza del tercer metatarsiano, y una menor carga en los metatarsianos más periféricos $\left(1^{\circ}\right.$ y $\left.5^{\circ}\right)$.

2. El grupo de pacientes con genu varo presenta en relación con los trastornos biomecánicos existentes en la extremidad afecta, un patrón de apoyo metatarsal diferente al de los sujetos normales, mostrando un hiperapoyo del cuarto y quinto metatarsianos y una disminución del apoyo soportado por los metatarsianos $1^{\circ}, 2^{\circ} \mathrm{y}$ $3^{\circ}$.

3. Tras la intervención quirúrgica se produce una realineación de la extremidad que origina una redistribución de las cargas metatarsales con aumento de la presión soportada por los metatarsianos $1^{\circ}, 2^{\circ}$ y $3^{\circ}$ y disminución de la carga soportada por los metatarsianos $4^{\circ}$ y $5^{\circ}$.

4. No existen diferencias significativas para el patrón de apoyo plantar en los individuos en que se consigue tras la artroplastia de rodilla un eje en valgo fisiológico $\left(4^{\circ}-10^{\circ}\right)$ y aquellos en los que no se logra esta corrección.

\section{Bibliografía}

1. LLANOS ALCAZAR, L.F.; ACEBES CACHAFEIRO, J.C.: "El pie". Monografías médico-quirúrgicas del aparato locomotor. Ed. Masson. Barcelona, 1997.

2. ABBOUD, R.J.; ROWLEY, D. Y.: "Foot pressure Measurement". In: Surgery of Disorders of the Foot and Ankle: 123-138. London, Martin Dunitz Limited, 1996:

3. DOMINGO, J; HERRERA, A; MARTÍNEZ, A; OLAVARRÍA, I, y PÉREZ GARCÍA, JM: "Contribución y límites de la baropodometría electrónica”. Rev Ortop Traumatol, 42(3): 189-192, 1998.

4. PEREZ GARCIA, J.M ${ }^{\text {a }}$; TABUENCA, A.; LOPEZ, J.E.; ORRITE, C.; MARTINEZ, J.; HERRERA, A.: "Estudio del apoyo metatarsal en bipedestación mediante podoscopio electrónico de alta resolución”. Biomecánica, IV, 6: 19-24, 1996.

5. VILADOT, R; COHI, O, y CLAVELL, S: Ortesis y prótesis del aparato locomotor. Masson, $\mathrm{S}$. A. Barcelona, 1987.

6. HSU, R.W.; HIMENO, S.; COVENTRY, M.B.; CHAO, E.: "Normal axial alignment of the lower extremity and load-bearing distribution at the knee". Clin Orthop, 255: 215-227, 1990.

7. MAQUET, P.G.J.: "Biomechanics of the knee". Springer-Verlag. Berlín, 1976.

8. MORELAND, J.R.; BASSETT, L.W.; HANKER, G.J.: "Radiographic analysis of the axial alignment of the lower extremity". J Bone Joint Surg, 69 A: 745-749, 1987.

9. TEW, M.; WAUGH, W.: "Tibiofemoral alignment and results of knee replacement". J Bone Joint Surg, 67B: 551-556, 1985.

10. BRUGIONI, D.J.; ANDRIACCHI, T.P.; GALANTE, J.O.: "A functional and radiographic analysis of the total condylar knee arthroplasty". $J$ Arthroplasty, 5, 2: 173-180, 1990.

11. LOTKE, P.A.; ECKER, M.L.: "Influence of positioning of prosthesis in total knee replacement". J Bone Joint Surg, 59 A: 77-79, 1977.

12. ANDRIACCHI, T.P.: "Functional analysis of pre and post-knee surgery: total knee arthroplasty and ACL reconstruction". J Biomech Eng, 115, 4B: 575$581,1993$.

13. ANDRIACCHI, TP: Biomechanics and gait analysis in total knee replacement. Orthopaedic Rewiew, 17(5): 470-473, 1988.

14. EDWARDS, E.; MILLER, J.; CHAN, K.H.: "The effect of postoperative collateral ligament laxity in total knee arthroplasty". Clin Orthop, 236: 44-51, 1988.

15. SMITH, J. W.: "The act standing". Acta Orthop Scand, V, 2: 159-176, 1953. 